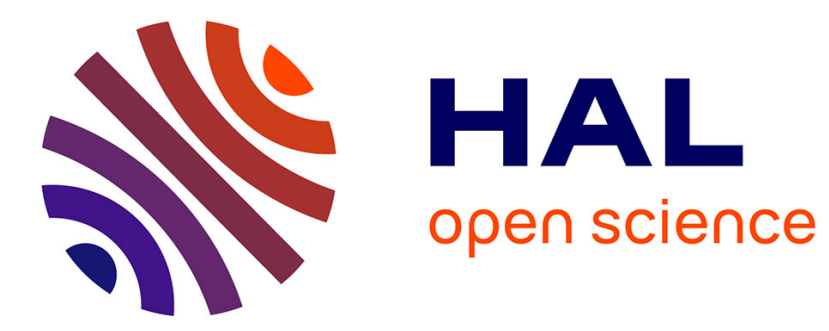

\title{
Fast and accurate intracorporeal targeting through an anatomical orifice exhibiting unknown behavior
}

Rémi Chalard, David Reversat, Guillaume Morel, Marie-Aude Vitrani

\section{To cite this version:}

Rémi Chalard, David Reversat, Guillaume Morel, Marie-Aude Vitrani. Fast and accurate intracorporeal targeting through an anatomical orifice exhibiting unknown behavior. ICRA, Jun 2020, Paris, France. hal-02863542

\section{HAL Id: hal-02863542 \\ https://hal.science/hal-02863542}

Submitted on 10 Jun 2020

HAL is a multi-disciplinary open access archive for the deposit and dissemination of scientific research documents, whether they are published or not. The documents may come from teaching and research institutions in France or abroad, or from public or private research centers.
L'archive ouverte pluridisciplinaire HAL, est destinée au dépôt et à la diffusion de documents scientifiques de niveau recherche, publiés ou non, émanant des établissements d'enseignement et de recherche français ou étrangers, des laboratoires publics ou privés. 


\title{
Fast and accurate intracorporeal targeting through an anatomical orifice exhibiting unknown behavior.
}

\author{
Rémi Chalard ${ }^{1}$, David Reversat ${ }^{1}$, Guillaume Morel $^{1}$, Marie-Aude Vitrani ${ }^{1}$
}

\begin{abstract}
Surgery may involve precise instrument tip positioning in a minimally invasive way. During these operations, the instrument is inserted in the body through an orifice. The movements of the instrument are constrained by interaction forces arising at the orifice level. The physical constraints may drastically vary depending on the patient's anatomy. This introduces uncertainties that challenge the positioning task for a robot. Indeed, it raises an antagonism: On one side, the required precision appeals for a rigid behavior. On the other side, forces applied at the entry point should be limited, which requires softness. In this paper we choose to minimize forces at the orifice by using a passive ball joint wrist to manipulate the instrument. From a control perspective, this leads to consider the task as a 3 DOF wrist center positioning problem, whose softness can be achieved through conventional low impedance control. However, positioning the wrist center, even with a high static precision, does not allow to achieve a high precision of the instrument tip positioning when the orifice behavior is not known. To cope with this problem, we implement a controller that servos the tip position by commanding the wrist position. In order to deal with uncertainties, we exploit an adaptive control scheme that identifies in real-time the unknown mapping between the wrist velocity and the tip velocity. Both simulations and in vitro experimental results show the efficiency of the control law.
\end{abstract}

\section{INTRODUCTION}

During minimally invasive surgery, instruments and imaging devices are inserted into a patient through small orifices. The orifice can be created by the surgeon, e.g. during laparoscopic surgery where cannulas are positioned through the abdominal wall. It can also be natural, e.g. during a prostate biopsy where an ultrasound probe and a needle are inserted through the patient's anus and rectum.

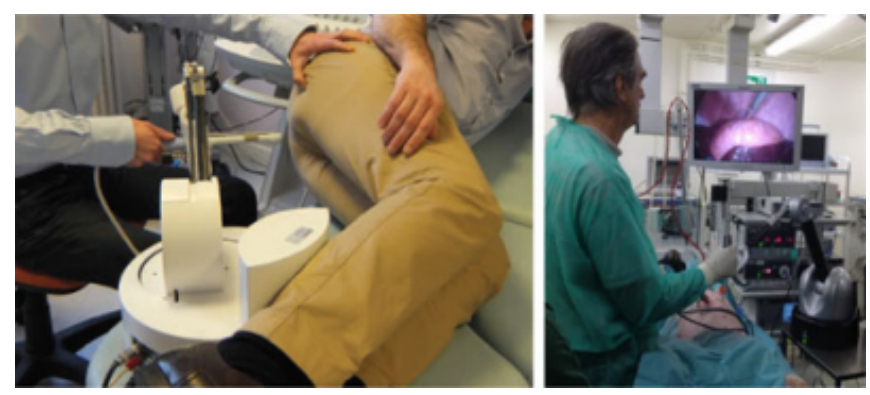

Fig. 1. Examples of keyhole surgery.

Inserting the instrument through an orifice induces mechanical constraints due to forces that appear at the instrument insertion area. For patient safety, these forces should

1 Sorbonne Université, INSERM U1150, CNRS UMR 7222, Institut des Systèmes Intelligents et de Robotique (ISIR) F-75005, Paris, France, [chalard, reversat, morel, vitrani] disir.upmc.fr be minimized during instrument manipulation. When a robot is used to manipulate an instrument for keyhole surgery, a kinematic solution is commonly used to achieve such a force minimization. Namely, the robot rigidly constrains the movements of the instrument along 4 degrees of freedom : one translation along the instrument axis and three rotations around a given point $R$. The model underlying this choice is that the body stiffness is maximal at an anatomical point $A$ located a few millimeters underneath the external body surface, while the rotational stiffness is low compared to the linear stiffness at point $A$. Therefore, minimizing the interaction forces at the orifice area is supposed to be solved by achieving $R=A$.

In the literature, two methods are used to rigidly constrain the instrument movements along 4 DOFs. In some cases, such as [1], a 4-DOF robot exhibiting a remote center of motion (RCM) is used. The robot base is placed in the workspace prior to instrument manipulation, in such a way that its RCM $R$ coincides with the insertion entry point $A$. In other cases, such as in [2], a fully actuated, 6-DOF robot is used and the kinematic constraint is achieved by the mean of robot control. This allows placing the robot base independently from the insertion point location as $R$ is not fixed with respect to the robot base. Note that in this case a registration of $A$ with respect to the robot base has to be done prior to the operation, as proposed in [3],[4], [5]. Alternatively, a force sensor can be used to identify on line point $A$ by minimizing the interaction forces, as proposed in [6]. A main limitation of all these approaches is that it rely on a model that does not always correspond to the reality: in many cases, minimizing the interaction forces at the entry port is not equivalent to perfectly pivoting around a fixed point. In a previous work, [7], we have shown that when urologists perform a prostate biopsy while minimizing the forces in the rectum it is not possible to define a fixed point $A$ around which the ultrasound probe rotates independently from the penetration depth. Therefore, minimizing $\|\overrightarrow{A R}\|$, either through installation, registration, or control, is not appropriate and may lead to non minimal forces at the insertion area.

In spite of rigidly constraining the robot to $4 \mathrm{DOF}$, the robot can also involve passive (unactuated) joints at the wrist. In [8], [9], [10], or [11], a combination of three active DOFs is used to position the wrist center; two passive joints in the wrist allows free orientation of the instrument axis, while the last DOF, corresponding to the instrument rotation around its axis, is motorized. Such a combination allows to reduce the degree of hyperstaticity and is likely to reduce forces at the 
insertion area: if a large lateral force is applied, due to the wrist passivity, the instrument axis orientation will change accordingly and the force will be lowered. Interestingly, this force minimization property does not depend on any knowledge on the fulcrum location with respect to the robot.

A main drawback occurs when a precise location is to be reached by the instrument tip $T$. Indeed, the position of $T$ inside the patient results not only from the position of the wrist center $W$, which is directly controllable by the three first actuators of the robot. Rather, the mechanical behavior of the insertion area also influences the tip position. In a previous paper, [7], we have evidenced this problem and proposed to identify the mapping between the wrist velocity, $\dot{W}$ and the tip velocity $\dot{T}$. This identification allowed to compute the desired displacement of point $W$ from the desired displacement at point $T$. However, this approach resulted in a very slow adaptation and remaining errors in the tip positioning, as a consequence of residual errors in the identified model.

In the present paper, we reformulate the control problem by a feedback at point $T$, add an on-line fast adaptation stage, and show a robust convergence and high precision through both simulations (involving very large errors in the initial model estimation) and experiments.

\section{ROBOTIC DEVICE AND ITS CONTROL LAW}

\section{A. Robotic device}

Apollo (Fig. 2), fits in the category of the free-wrist robots, [12].

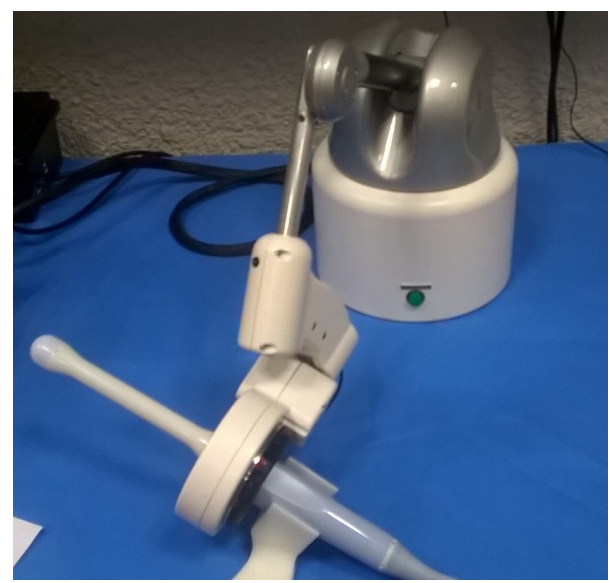

Fig. 2. Apollo robot V2.

Apollo comprises six pivot joints serially assembled according to a conventional anthropomorphic geometry: the three first active joints form the shoulder and the elbow while the wrist is composed of the three last passive joints, whose axes coincide at point $W$. The kinematics is sketched in Fig.3.

Kinematic models mapping joint positions $q$ (resp. velocities $\dot{q}$ ) into point $W$ and point $T$ positions (resp. velocities $\dot{W}$ and $\dot{T}$ ) follows directly from the Denavit and Hartenberg parameters given in Table I, by using the method described in [13].

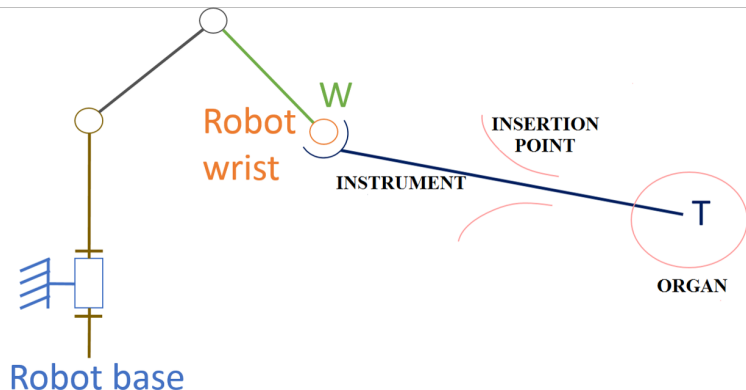

Fig. 3. Apollo kinematics

TABLE I

DH PARAMETERS OF THE APOLLO ROBOT.

\begin{tabular}{|c||c|c|c|c|}
\hline $\mathrm{i}$ & $\alpha_{i}$ & $a_{i}$ & $\theta_{i+1}$ & $d_{i+1}$ \\
\hline 0 & 0 & 0 & $\theta_{1}$ & 0 \\
\hline 1 & $\pi / 2$ & 0 & $\theta_{2}$ & 0 \\
\hline 2 & 0 & $25 \mathrm{~cm}$ & $\theta_{3}$ & 0 \\
\hline 3 & $\pi / 2$ & 0 & $\theta_{4}$ & $30 \mathrm{~cm}$ \\
\hline 4 & $-\pi / 4$ & 0 & $\theta_{5}$ & 0 \\
\hline 5 & $-\pi / 2$ & 0 & $\theta_{6}$ & 0 \\
\hline
\end{tabular}

\section{B. Low level controller}

Apollo offers 2 modes of operation:

- The free mode, characterized by high transparency and gravity compensation. This allows for manually positioning the probe under ultrasound guidance [14].

- The locked mode, during which the urologist has his/her hands free to perform the needle placement and the biopsy. Here, it is desired that the robot maintains precisely the target position, [14].

The control law used for the locked mode is an impedance controller generating forces in response to position errors. Due to the passive wrist, the force transmission model at point $W$ writes:

$$
\tau=\mathbf{J}_{r}^{\mathrm{T}} \mathbf{f} .
$$

where $\tau=\left[\begin{array}{lll}\tau_{1} & \tau_{2} & \tau_{3}\end{array}\right]^{\mathrm{T}}$ is the vector of the 3 first motors joint torques, $\mathbf{f}$ the equivalent force at point $W$ and $\mathbf{J}_{r}$ the jacobian matrix mapping the velocity of the three first robot joints into point $W$ velocity:

$$
\dot{W}=\mathbf{J}_{r}\left[\begin{array}{lll}
\dot{q}_{1} & \dot{q}_{2} & \dot{q}_{3}
\end{array}\right]^{\mathrm{T}}
$$

The control law writes:

$$
\tau=\tau_{\text {grav }}+\mathbf{J}_{r}^{\mathrm{T}}\left(k_{d} \varepsilon_{W}+k_{i} \int_{0}^{t} \varepsilon_{W} d u\right)
$$

where $\tau_{\text {grav }}$ is the gravity compensation [12], $\varepsilon_{W}=W_{d}-W$ is the error between the desired and the current position of the robot wrist, $k_{d}$ and $k_{i}$ are respectively the proportional and the integral gains, both scalar. Choosing low values for 
$k_{p}$ and $k_{i}$ allows for exhibiting a low stiffness at point $W$, yet with a null static error (slow cancellation of the errors despite disturbances at the insertion point).

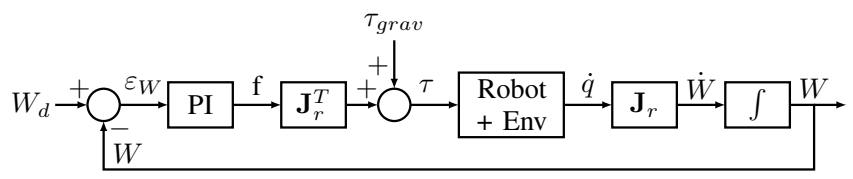

Fig. 4. Apollo control law at point $\mathrm{W}$

The present paper concerns the development of a third operation mode: adjust. Here the robot shall automatically displace the probe tip toward a desired location. The urologist will use this mode to realize small adjusting displacements with high precision if $s /$ he is not satisfied by the result of the manual positioning realized in the free mode. S/he will typically generate from an interface a desired displacement of the tip, $\Delta T$. How to precisely an stably realize $\Delta T$ at the tip level in spite of uncertainties in the behavior of the insertion zone behavior, this is the question under study.

\section{CONTROLling THE TIP POSITION}

\section{A. Open-loop and closed-loop tip position control}

During a surgical operation, the objective is not to position the robot wrist center $W$ but rather to control the position of the instrument tip $T$. To reach a desired location $T_{d}$, a first option consists in computing the corresponding desired position $W_{d}$ for the wrist center. To do so, we first assume that, locally, the mapping between $\dot{W}$ and $\dot{T}$ writes:

$$
\dot{T}=\mathbf{J} \dot{W} .
$$

Assuming that, when using the adjust mode, the urologist specifies a displacement $\Delta T$ for the tip while the position of the wrist is $W_{0}$, a desired position for the control law (3) can be computed by:

$$
W_{d} \approx W_{0}+\hat{\mathbf{J}}^{-1} \Delta T,
$$

where $\hat{\mathbf{J}}$ is an estimate of $\mathbf{J}$.

However, this approach is very sensitive to errors made in estimating $\mathbf{J}$, as the error at point $T$ is not servoed. To overcome this issue, one can equip the passive joints of the spherical wrist with position sensors, allowing for computation of point $T$ location in real-time. Closing the loop at point $T$ is then possible with the following control law:

$$
\tau=\tau_{\text {grav }}+\mathbf{J}_{r}^{\mathrm{T}}\left(k_{d} \hat{\mathbf{J}}^{-1} \varepsilon_{T}+k_{i} \int_{0}^{t} \hat{\mathbf{J}}^{-1} \varepsilon_{T} d u\right)
$$

where $\varepsilon_{T}:=T_{d}-T$, as illustrated in Fig. 5. With such a controller, due to the integration of $\varepsilon_{T}$, a null error at point $T$ is guaranteed providing that the system remains stable.

\section{B. Simulation results}

In order to illustrate the behavior of controllers described by Eq. (3)-(5) and Eq. (6), a simple situation is considered through a simulated example: the instrument insertion is

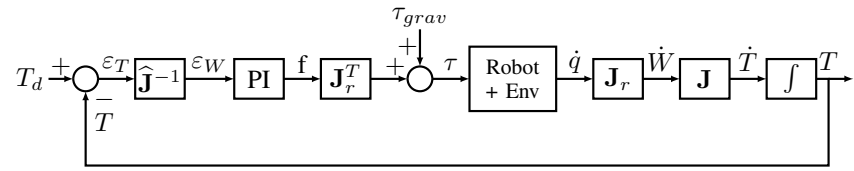

Fig. 5. Closed-loop control of the instrument tip

made through a known fixed point. A conventional lever model allows to derive $\mathbf{J}$ :

$$
\hat{\mathbf{J}}=\left(\begin{array}{ccc}
-\frac{p}{(L-p)} & 0 & 0 \\
0 & 1 & 0 \\
0 & 0 & -\frac{p}{(L-p)}
\end{array}\right)
$$

where $L=\|\overrightarrow{W T}\|$ and $d$ is the distance from $T$ to the fixed insertion point, see Fig. 6.

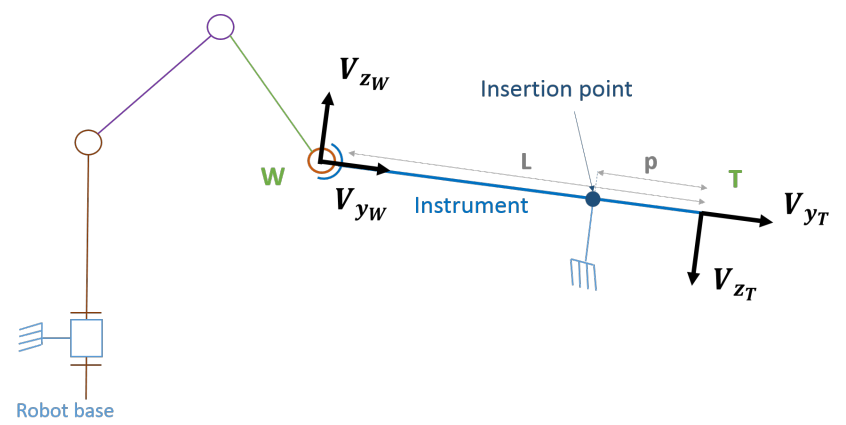

Fig. 6. A simplistic lever model around a fixed point.

A first simulation result is given in Fig. 7. Here, the interaction matrix is:

$$
\mathbf{J}=\left(\begin{array}{ccc}
-0.5 & 0 & 0 \\
0 & 1 & 0 \\
0 & 0 & -0.5
\end{array}\right)
$$

Moreover, a perfect knowledge of the interaction matrix is assumed: $\hat{\mathbf{J}}=\mathbf{J}$.

It can be seen in Fig. 7 that in this ideal situation, both controllers behave equivalently. A desired displacement $\Delta T$ of $10 \mathrm{~mm}$ in a direction that is perpendicular to the insertion axis is commanded and realized with a final null error. Notice that the small overshoot observed in the plots is due to the robot dynamics and integrator effect.

In a second simulation, $\mathbf{J}$ being unchanged, an estimation error is introduced:

$$
\hat{\mathbf{J}}=\left(\begin{array}{ccc}
-1 & 0 & 0 \\
0 & 1 & 0 \\
0 & 0 & -1
\end{array}\right) .
$$

The simulation results, displayed in Fig. 8, show that:

- with the controller expressed at point $W$, the final error is not zero, as expected;

- with the controller expressed at point $T$, the error converges toward zero but the transient dynamics is changed (a higher peak response can be observed). This is due to the imperfect decoupling realized by $\hat{\mathbf{J}} \neq \mathbf{J}$. 

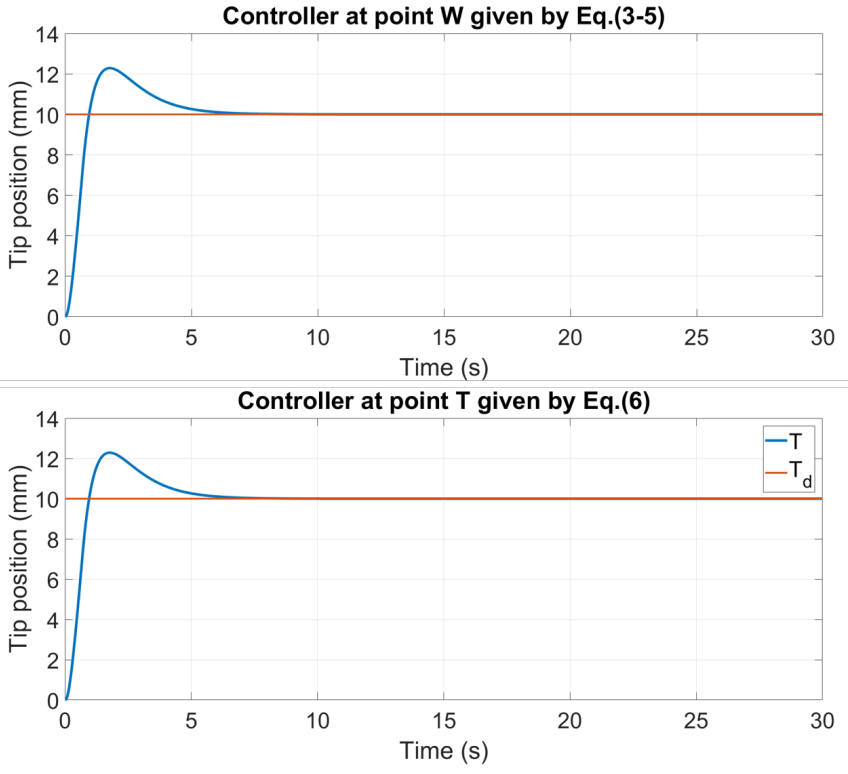

Fig. 7. Simulation results at point $T$ for both controllers, when $\hat{\mathbf{J}}=\mathbf{J}$
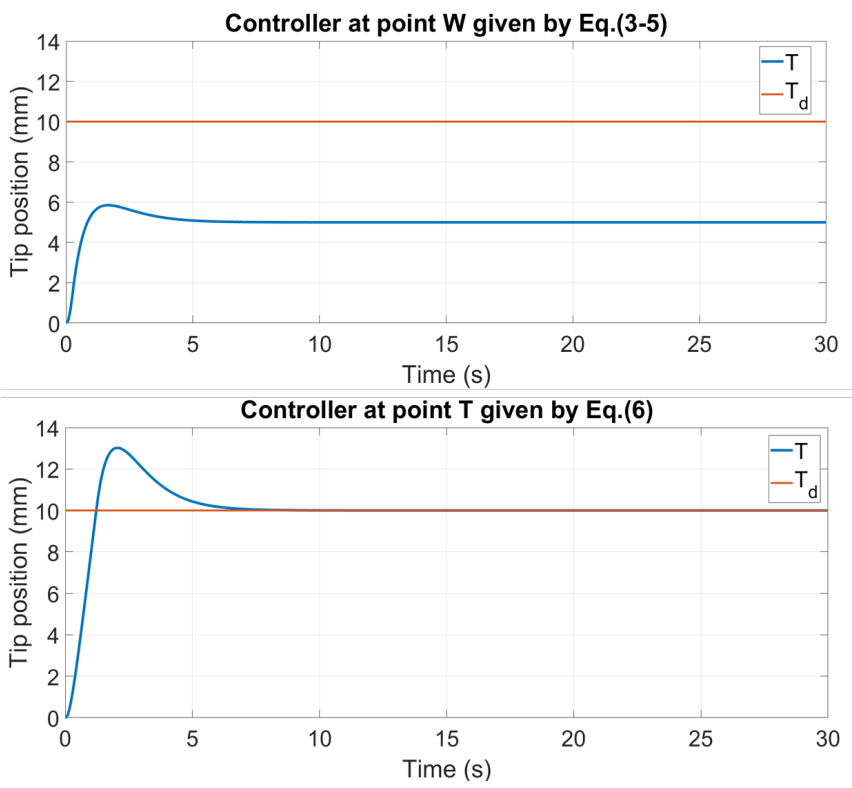

Fig. 8. Simulation results at point $T$ for both controllers, when $\hat{\mathbf{J}} \neq \mathbf{J}$

To further illustrate the degradation of the closed loop dynamics with Controller (6), a last simulation is provided. Here :

$$
\hat{\mathbf{J}}=\left(\begin{array}{lll}
1 & 0 & 0 \\
0 & 1 & 0 \\
0 & 0 & 1
\end{array}\right)
$$

This simulates a situation where, the estimated model is completely wrong. Instead of representing a fixed point around which the instrument can rotate, the estimated model assumes that the orientation of the instrument is maintained constant by the surrounding tissues while the translations perpendicular to the instrument axis are free. With such a drastic estimation error, without surprise, the closed loop behavior becomes unstable as a positive feedback is implemented, see Fig. 9.

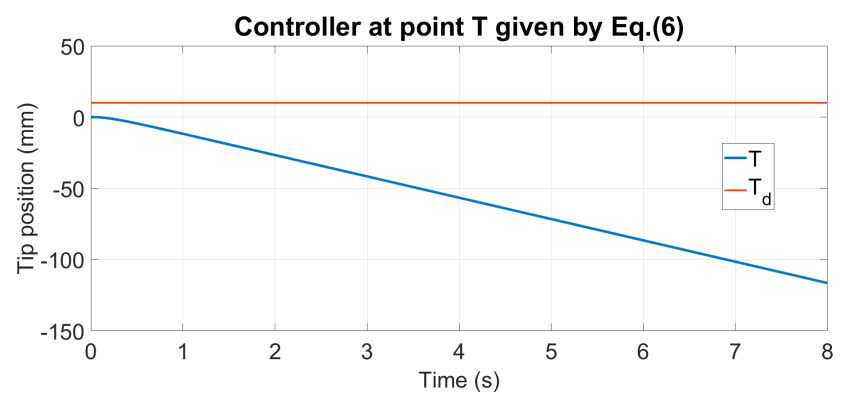

Fig. 9. When the estimation error becomes large, controller (6) may diverge.

\section{ADAPTIVE CONTROL}

\section{A. Problem definition}

As emphasized in Sec.III-B, errors in the estimation of the instrument-patient interaction lead to deteriorate the closed loop behavior when point $T$ is fed back to the controller. This can have a significant impact in real situations, where the interaction cannot be modeled as a fulcrum and not be precisely known in advance. For example, in [7], it has been shown that the insertion point can be displaced more than $20 \mathrm{~mm}$ during a prostate biopsies session. In [15] it is demonstrated that the manipulation of the uterus with an instrument through the vagina during a surgery can not be modeled as a fulcrum.

In fact, the mapping $\mathbf{J}$ from $W$ displacements to $T$ displacements depends on how the tissues surrounding the insertion site deform. As the tissues deformation can not be modeled and varies according to the site insertion (anus, stomach, uterus, ...) the interaction between $\dot{W}$ and $\dot{T}$ can be modelled with the general form:

$$
\mathbf{J}=\left(\begin{array}{lll}
\mathbf{J}_{x x} & \mathbf{J}_{x y} & \mathbf{J}_{x z} \\
\mathbf{J}_{y x} & \mathbf{J}_{y y} & \mathbf{J}_{y z} \\
\mathbf{J}_{z x} & \mathbf{J}_{z y} & \mathbf{J}_{z z}
\end{array}\right)
$$

Moreover, in order to take the tissues deformation into account during a manipulation of the instrument, $\mathbf{J}$ has to be continuously estimated as $\hat{\mathbf{J}}$.

In a previous work, [7], we partially solved the problem assuming that the structure of $\mathbf{J}$ can be simplified. Indeed, basing on the fact that the instrument is supposed to be rigid, the displacements of $W$ are supposed to be equal to those of $T$ along the penetration axis $y$. One thus estimates $\mathbf{J}$ by a least square optimization thanks to the recording of the velocity of the robot wrist $(\dot{W})$ and the instrument tip $(\dot{T})$.

In a more general case described by Eq. (11), it appears that an instantaneous measurement of $\dot{W}$ and $\dot{T}$ are not sufficient to identify the 9 unknown elements of $\mathbf{J}$ as only three equations are available. 


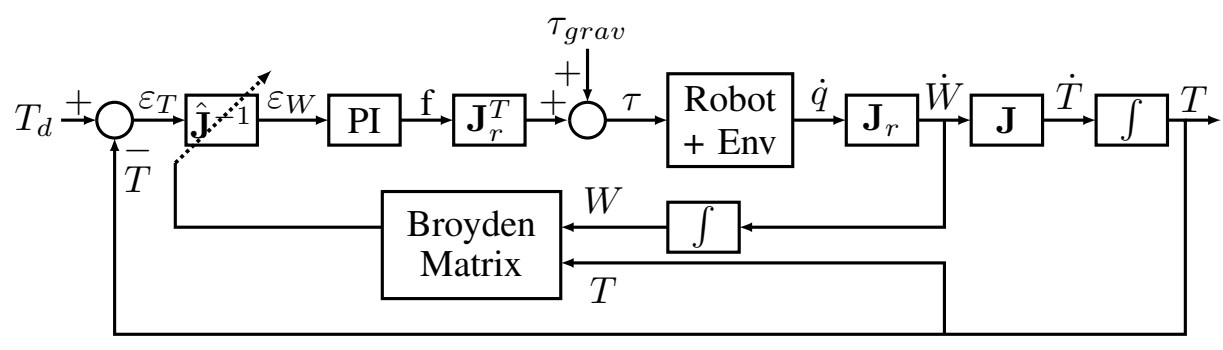

Fig. 10. Closed loop control of the instrument tip with the interaction matrix $\hat{\mathbf{J}}$ estimated thanks to the Broyden method

\section{B. Broyden algorithm}

The problem stated before is a nonlinear problem of optimization since the matrix $\mathbf{J}$ may depend on the position $W$ and on the ill-known environment. In the literature, one of the most common numerical method to solve this kind of problem is the Newton method. More especially, when it is necessary to estimate a Jacobian matrix, the Broyden's method applies. It is an iterative method that can be used to estimate the Jacobian matrix [16]. It uses an initial guess and then generates a sequence of approximate solutions. In practice, it provides good convergence results providing that the initial value is not too far from the real value. Interestingly, this method has a low computational cost allowing for an online estimation.

With the Broyden method [17], the estimated matrix $\hat{\mathbf{J}}_{k}$ at a discrete time $k$ is computed thanks to:

$$
\hat{\mathbf{J}}_{k}=\hat{\mathbf{J}}_{k-1}+\alpha \frac{\delta T_{k}-\hat{\mathbf{J}}_{k-1} \cdot \delta W_{k}}{\left\|\delta W_{k}\right\|^{2}} . \delta W_{k}^{T},
$$

where:

- $\delta T_{k}=T_{k}-T_{k-1}$ is the measured displacement of $T$ between the two iterations, in the robot frame.

- $\delta W_{k}=W_{k}-W_{k-1}$ is the measured displacement of $W$ between the two iterations, in the robot frame.

- $\alpha$ is a scalar factor generally chosen between 0 and 1 .

The parameter $\alpha$ tunes the convergence speed. When tuning this parameter, a compromise must be achieved between increasing convergence speed (with high values) and increasing robustness to noise (with low values).

Also, if the input data variation is too small or null, the computation may become unstable. To avoid this instability, on needs to verify that:

$$
\delta W_{k}^{\mathrm{T}} . \delta W_{k}=\left\|\delta W_{k}\right\|^{2}>0
$$

A threshold is introduced to ensure the previous condition is verified.

$$
\begin{aligned}
\text { If: } & \left\|\delta W_{k}\right\| \leq r_{\varepsilon} \\
\text { then: } & \hat{\mathbf{J}}_{k}=\hat{\mathbf{J}}_{k-1}
\end{aligned}
$$

where $r_{\varepsilon}$ is a threshold to be tuned depending on the application.

For both the simulations and experiments, $\alpha$ and $r_{\varepsilon}$ has been defined as:

- $\alpha=0.5$,
- $r_{\varepsilon}=0.1 \mathrm{~mm}$.

The final control scheme is given in Fig. 10

\section{Simulation results}

In order to evaluate the capacity of the adaptive controller to deal with large uncertainties, a simulation is performed with the situation depicted by Eq. (10) and Eq. (8).

The simulation result is plotted in Fig. 11, and has to be compared with the simulation result depicted in Fig. 9, in a non adaptive case. While the initial behavior tends to diverge $(0<T<3.5 \mathrm{~s})$, the on-line estimation changes leading to a convergence with a null error.

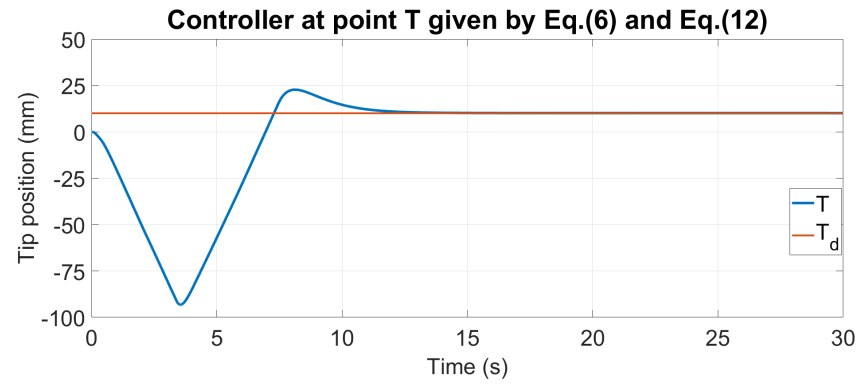

Fig. 11. Simulation of a reaching task at point $T$ with the adaptive controller and a very large initial estimation error.

Furthermore, even with a strongly wrong initial matrix, after the convergence, the Broyden method provide the final estimation:

$$
\hat{\mathbf{J}}=\left(\begin{array}{ccc}
-0.642 & -0.191 & 0.0089 \\
0 & 1 & 0 \\
-0.0629 & 0.0773 & -0.538
\end{array}\right)
$$

Notice that although $\hat{\mathbf{J}}$ differs from $\mathbf{J}$, the estimation error is small enough to obtain convergence.

\section{EXPERIMENTAL SET-UP AND RESULTS}

In order to validate the performances of the two controllers developed in the previous section (Sec.IV-C), both of them are implemented in the robotic device : Apollo [12]. Moreover, to reproduce an orifice, the instrument is inserted through a clamping jaw cover with foam (see Fig.12).

The foam on the clamping jaw allow to emulate an unknown interaction between the instrument and the tissues. The insertion point around which the instrument is supposed to turn can move within an ellipsoid $2 \mathrm{~cm}$ long. It can be 


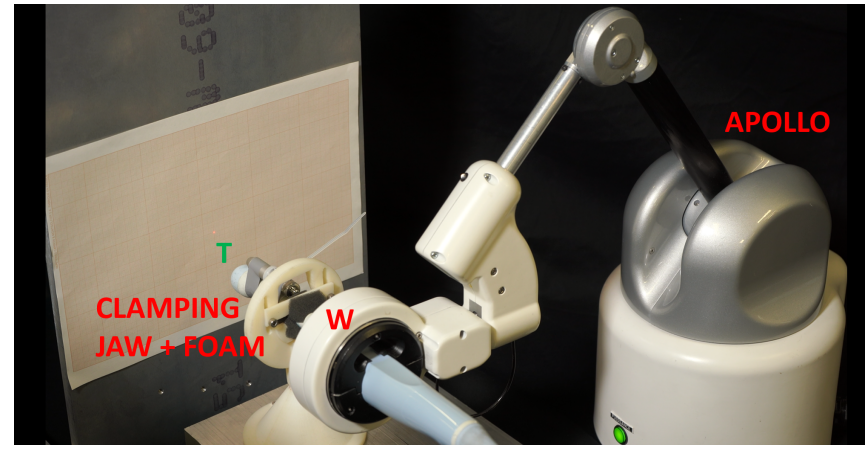

Fig. 12. Description of the experimental set-up with the robot and the interface.

assimilated to the elasticity of the human tissues at the insertion point.

The experiment is a set of 20 trials for both controller with and without adaption as defined in Fig.5 and Fig.10. Each trial consists in reaching a target $20 \mathrm{~mm}$ from different initial position of point $T$. The estimated matrix $\widehat{\mathbf{J}}$ is manually estimated from a rough measurement on the insertion length:

$$
\hat{\mathbf{J}}=\left(\begin{array}{ccc}
-1.2 & 0 & 0 \\
0 & 1 & 0 \\
0 & 0 & -1.2
\end{array}\right)
$$

The same matrix is used for both the controller. For the first controller without adaptation, it stays the same $\hat{\mathbf{J}}$ during all the displacement. For the second controller with adaptation (see Fig.10), it is implemented as the initial value of the matrix $\hat{\mathbf{J}}$.

The position of points $T$ and $W$ are registered during each trial. The norm of $T-T_{0}$ where $T_{0}$ is the initial position of point $T$ is computed for each trial. Figures 13 and 14 shows obtained results.

These results are noisy because of the dynamics of the system. Even though, the desired position is reached whatever controller is used, as explain Sec.IV-C.

However, the Broyden based controller is more efficiency than the controller without adaption. Indeed, the convergence to the target is faster (5 times faster based on the 40 trials) as shown in Fig.15. Moreover, the displacement is smoother and more precise. It is due to the better estimation of the

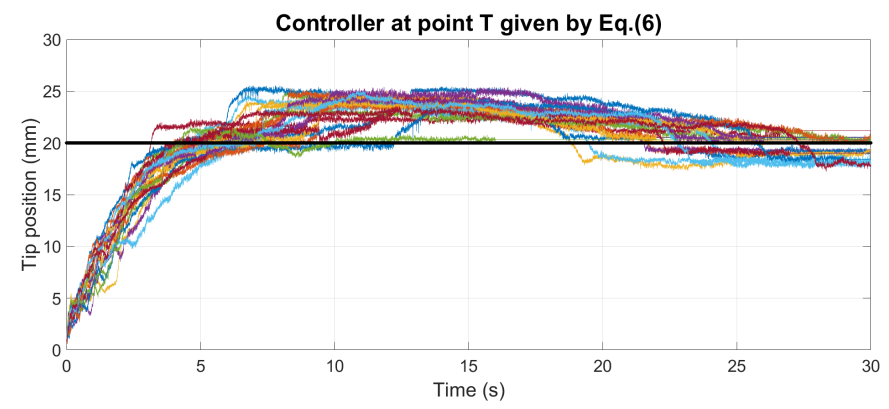

Fig. 13. Set of 20 displacements of $20 \mathrm{~mm}$ for controller without adaptation, as defined in Fig.5

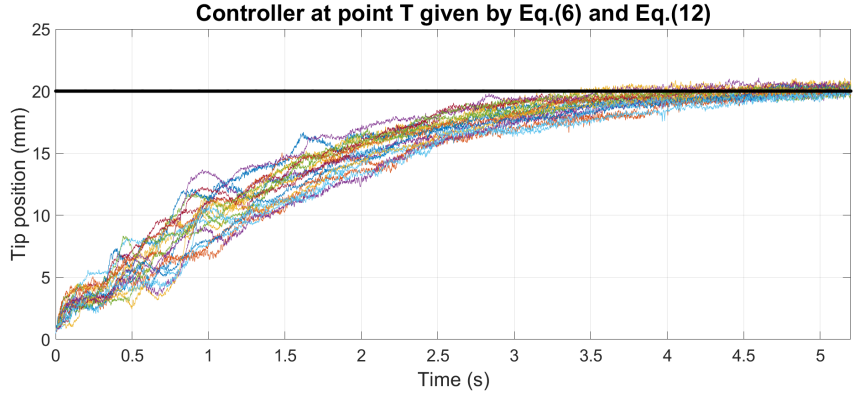

Fig. 14. Set of 20 displacements of $20 \mathrm{~mm}$ for controller with adaptation as defined in Fig.10

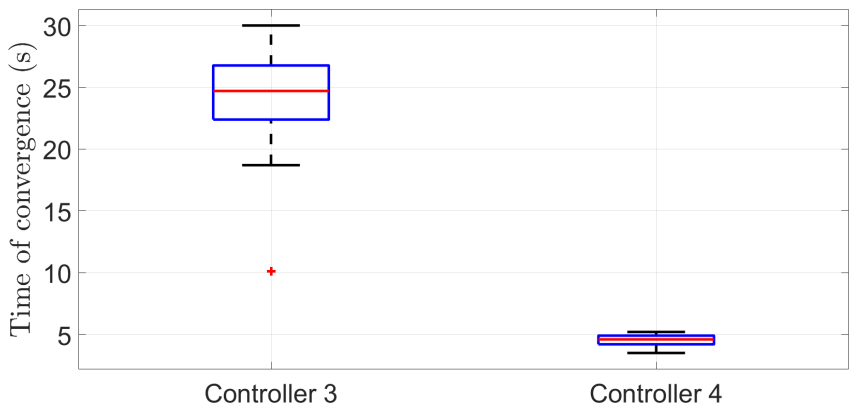

Fig. 15. Time of convergence for the 40 trials (Controller 3: without adaptation - Controller 4: with adaptation

interaction matrix thanks to the Broyden estimator. Indeed, in a perfect case, the error should exponentially decreased due to the PID controller.

\section{CONCLUSION}

In this paper, authors present a new control law for a 6 DOF's robot with a passive wrist to automatically reach a defined target with a tool inserted through an orifice which mechanical behavior is unknown. From a control perspective, this leads to consider the task, at the lowest level, as a 3 DOF wrist center positioning problem. However, positioning the wrist center, even with a high static precision, does not allow to achieve a high precision of the instrument tip positioning when the orifice behavior is not known. To cope with this problem, we implement a controller that servos the tip position by commanding the wrist position. In order to deal with uncertainties, we exploit an adaptive control scheme that identifies in real-time the unknown mapping between the wrist velocity and the tip velocity. Both simulations and in vitro experimental results show the efficiency of the proposed control law. Future work will focus on reaching a moving target. Indeed, the movement of the instrument and the body wall may induce organ's deformation leading to a displacement of the target (this is the case during prostate biopsy). The main idea is to use the proposed control scheme in a visual servoing loop based onto a real time measurement of the target position in the medical images. 


\section{ACKNOWLEDGMENT}

This work was supported by the French state funds managed by the ANR (Agence Nationale de la Recherche) within the Invesissements dAvenir Program (Labex CAMI) under Reference ANR-11-LABX-0004.

\section{REFERENCES}

[1] G. Guthart and J. Salisbury, "The intuitive telesurgery system : Overview and application," Proc IEEE International Conference on Robotics and Automation, 2000, pp. 618-621, 2000. [Online]. Available: http://ci.nii.ac.jp/naid/20001085379/en/

[2] R. Konietschke, U. Hagn, M. Nickl, S. Jörg, A. Tobergte, G. Passig, U. Seibold, L. Le-Tien, B. Kübler, M. Gröger, F. Fröhlich, C. Rink, A. Albu-Schäffer, M. Grebenstein, T. Ortmaier, and G. Hirzinger, "The dlr mirosurge - a robotic system for surgery," in IEEE International Conference on Robotics and Automation, 2009, pp. 1589-1590. [Online]. Available: http://elib.dlr.de/76870/

[3] C. D. Pham, F. Coutinho, A. C. Leite, F. Lizarralde, P. J. From, and R. Johansson, "Analysis of a moving remote center of motion for robotics-assisted minimally invasive surgery," in 2015 IEEE/RSJ International Conference on Intelligent Robots and Systems (IROS), Sept 2015, pp. 1440-1446.

[4] E. M. Boctor, R. J. W. III, H. Mathieu, A. M. Okamura, and G. Fichtinger, "Virtual remote center of motion control for needle placement robots," Computer Aided Surgery, vol. 9, no. 5, pp. 175183, 2004.

[5] E. Dombre, M. Michelin, F. Pierrot, P. Poignet, P. Bidaud, G. Morel, T. Ortmaier, D. Sallé, N. Zemiti, P. Gravez, M. Karouia, and N. Bonnet, "Marge project: Design, modeling and control of assistive devices for minimally invasive surgery," in Medical Image Computing and Computer-Assisted Intervention - MICCAI 2004: 7th International Conference, Saint-Malo, France, September 26-29, 2004. Proceedings, Part II, C. Barillot, D. R. Haynor, and P. Hellier, Eds. Berlin, Heidelberg: Springer Berlin Heidelberg, 2004, pp. 1-8. [Online]. Available: https://doi.org/10.1007/978-3-540-30136-3-1

[6] A. Krupa, G. Morel, and M. de Mathelin, "Achieving high precision laparoscopic manipulation through adaptive force control," in Proceedings 2002 IEEE International Conference on Robotics and Automation (Cat. No.02CH37292), vol. 2, May 2002, pp. 1864-1869 vol.2.

[7] R. Chalard, D. Reversat, G. Morel, P. Mozer, and M.-A. Vitrani, "Precisely positioning the tip of an instrument inserted through an orifice with a free wrist robot: application to prostate biopsies," International Journal of Computer Assisted Radiology and Surgery, Feb 2018. [Online]. Available: https://doi.org/10.1007/s11548-0181718-6

[8] S. C. Low and L. Phee, "A review of master-slave robotic systems for surgery," in IEEE Conference on Robotics, Automation and Mechatronics, 2004., vol. 1, Dec 2004, pp. 37-42 vol.1.

[9] J. M. Sackier and Y. Wang, "Robotically assisted laparoscopic surgery," Surgical Endoscopy, vol. 8, no. 1, pp. 63-66, Jan 1994. [Online]. Available: https://doi.org/10.1007/BF02909496

[10] T. Ortmaier and G. Hirzinger, "Cartesian control issues for minimally invasive robot surgery," in Proceedings. 2000 IEEE/RSJ International Conference on Intelligent Robots and Systems (IROS 2000) (Cat. No.00CH37113), vol. 1, 2000, pp. 565-571 vol.1.

[11] V. F. Munoz, C. Vara-Thorbeck, J. G. DeGabriel, J. F. Lozano, E. Sanchez-Badajoz, A. Garcia-Cerezo, R. Toscano, and A. JimenezGarrido, "A medical robotic assistant for minimally invasive surgery," in Proceedings 2000 ICRA. Millennium Conference. IEEE International Conference on Robotics and Automation. Symposia Proceedings (Cat. No.00CH37065), vol. 3, April 2000, pp. 2901-2906 vol.3.

[12] C. Poquet, P. Mozer, G. Morel, and M.-A. Vitrani, "A novel comanipulation device for assisting needle placement in ultrasound guided prostate biopsies." IEEE, Nov. 2013, pp. 4084-4091. [Online]. Available: http://ieeexplore.ieee.org/document/6696941/

[13] J. Denavit and R. S. Hartenberg, "A kinematic notation for lower-pair mechanisms based on matrices." Trans. of the ASME. Journal of Applied Mechanics, vol. 22, pp. 215-221, 1955. [Online]. Available: http://ci.nii.ac.jp/naid/10008019314/en/
[14] C. Poquet, P. Mozer, M.-A. Vitrani, and G. Morel, "An Endorectal Ultrasound Probe Comanipulator With Hybrid Actuation Combining Brakes and Motors," IEEE/ASME Transactions on Mechatronics, vol. 20, no. 1, pp. 186-196, Feb. 2015. [Online]. Available: http://ieeexplore.ieee.org/document/6804035/

[15] J. D. Smet, J. Deprest, A. Thys, and E. V. Poorten, "In-vivo force sensing during laparoscopic sacrocolpopexy vaginal vault manipulation," 2018. [Online]. Available: http://dx.doi.org/10.21227/tqyc-d305

[16] N. Mansard, M. Lopes, J. Santos-Victor, and F. Chaumette, "Jacobian learning methods for tasks sequencing in visual servoing," in 2006 IEEE/RSJ International Conference on Intelligent Robots and Systems, Oct 2006, pp. 4284-4290.

[17] C. G. Broyden, "A class of methods for solving nonlinear simultaneous equations," Math. Comp. 19 (1965), pp. 577-593. 